\title{
Jumonji domain-containing protein 6 functions as a marker of head and neck squamous cell carcinoma at advanced stage with no effect on prognosis
}

\author{
BING GUO*, LEI WANG* , XINGJUN QIN, YI SHEN and CHUNYUE MA \\ Department of Oral and Maxillofacial, Head and Neck Oncology, 9th People's Hospital, \\ Shanghai Jiao Tong University School of Medicine, Shanghai Key Laboratory of Stomatology, Shanghai 200011, P.R. China
}

Received March 1, 2019; Accepted August 16, 2019

DOI: $10.3892 / \mathrm{ol} .2019 .10938$

\begin{abstract}
A number of studies have reported the upregulation and functional roles of Jumonji domain-containing protein 6 (JMJD6) in various types of cancer. However, little is known regarding the clinical significance of JMJD6 in head and neck squamous cell carcinoma (HNSCC), particularly in terms of large-cohort data. In the present study, bioinformatics analysis was performed, using the University of California Santa Cruz Xena Browser and the Gene Expression Profiling Interactive Analysis 2 server, based on the Cancer Genome Atlas HNSCC cohort. In addition, a validation cohort was constructed based on 98 HNSCC cases. JMJD6 overexpression and knockdown, colony-formation, Transwell and cell viability assays were performed. JMJD6 was highly expressed in HNSCC samples and was associated with advanced pathological stage. However, no significant association was observed between JMJD6 expression levels and overall survival or disease-free survival times of patients with HNSCC. Subsequent in vitro assays indicated that overexpression of JMJD6 promoted malignant progression of HNSCC, by regulating epithelial-mesenchymal transition. Nevertheless, JMJD6 overexpression had no significant effects on the viability of HNSCC cells treated with 5-fluorouracil or cisplatin. Thus, it can be concluded that JMJD6 may function as a marker of HNSCC at advanced stage, however with no effect on drug resistance or prognosis.
\end{abstract}

Correspondence to: Dr Chunyue Ma or Dr Yi Shen, Department of Oral and Maxillofacial, Head and Neck Oncology, 9th People's Hospital, Shanghai Jiao Tong University School of Medicine, Shanghai Key Laboratory of Stomatology, 639 Zhi Zao Ju Road, Shanghai 200011, P.R. China

E-mail: maxifama@163.com

E-mail: shenyi_777@126.com

*Contributed equally

Key words: Jumonji domain-containing protein 6, epithelialmesenchymal transition, head and neck squamous cell carcinoma, malignant progression, prognosis
For patients with advanced HNSCC and high JMJD6 expression, rational chemotherapy may be more beneficial than radical surgery, considering their quality of life.

\section{Introduction}

Jumonji domain-containing protein 6 (JMJD6) is a member of the Jumonji $\mathrm{C}$ domain-containing metalloenzymes $(1,2)$. For the past 17 years, studies have indicated that JMJD6 as a multidimensional protein in biological processes that interacts with multiple protein substrates in distinct molecular signaling pathways [such as U2 small nuclear RNA auxiliary factor 2 and p21 (RAC1) activated kinase 1] $(3,4)$. According to the current understanding, JMJD6 acts as either a histone demethylase, which removes methyl moieties on histone $\mathrm{H} 3$ at arginine 2 and $\mathrm{H} 4$ at arginine 3, or as a lysyl hydroxylase that targets the RNA splicing factor U2 small nuclear RNA auxiliary factor $2(2,5,6)$. In summary, JMJD6 serves diverse roles at the transcriptional, splicing, posttranscriptional and biochemical levels (7).

The upregulation and functional role of JMJD6 in cancer areas have been recently demonstrated (8). In breast cancer, overexpression of JMJD6 has been reported to be associated with poorer outcomes and more aggressive characteristics, via modulating cell proliferation and motility $(3,7)$. In hepatocellular carcinoma, JMJD6 promotes progression of malignancy via regulation of CDK4 by directly targeting its promoter (9). In melanoma, a positive feedback loop (JMJD6- serine/threonine-protein kinase PAK 1-mitogen-activated protein kinase 1-JMJD6) associated with the promotion of melanogenesis, cell proliferation, invasion and angiogenesis has been investigated (10). In oral squamous cell carcinoma (OSCC), JMJD6 has been demonstrated to be a novel regulator of cancer stem cells via upregulation of interleukin 4 expression (11).

However, little is known regarding the clinical significance of JMJD6 in head and neck squamous cell carcinoma (HNSCC), particularly in terms of large-cohort data. The Cancer Genome Atlas (TCGA) project provides an opportunity for the comprehensive understanding of human cancer, based on powerful and detailed information from a large cohort (12-14). In the present study, bioinformatics analysis was conducted using two online tools, the University of 
California Santa Cruz (UCSC) Xena Browser and Gene Expression Profiling Interactive Analysis 2 (GEPIA2), based on the TCGA primary HNSCC cohort data. The expression patterns, clinical significance and biological roles of JMJD6 in HNSCC were systematically illustrated based on the TCGA cohort and a validation cohort. Subsequent in vitro assays were performed to investigate the biological roles of JMJD6 in HNSCC.

\section{Materials and methods}

Bioinformatics analysis and validation. Bioinformatics analysis was performed based on the TCGA HNSCC cohort using the web-based tools GEPIA2 (http://gepia2.cancer-pku.cn) and the UCSC Xena Browser (http://xena.ucsc.edu) (12-14). Only cases of primary HNSCC were included in further analyses of the expression patterns of $J M J D 6$, snail family transcriptional repressor 1 (SNAII), vimentin (VIM), twist family bHLH transcription factor 1 (TWIST1), cadherin $2(C D H 2)$ and cadherin 1 $(\mathrm{CDH1})$. Heatmaps of defined gene sets were generated online (https://xenabrowser.net/heatmap), and detailed data were downloaded for further analysis (12-14).

A retrospectively validation HNSCC cohort was constructed, based on 98 primary HNSCC cases admitted to the Department of Oral Maxillofacial-Head and Neck Oncology, Shanghai 9th People's Hospital between January 2010 and December 2017. The clinicopathological characteristics of each case were collected for further analysis. All patients involved in the present study provided written informed consent, and the study was approved by the Medical Ethics Committee of the 9th People's Hospital, Shanghai Jiao Tong University School of Medicine (Shanghai, China) (SH9H-2019-0421).

Immunohistochemistry staining and scoring. Tissues were fixed in $10 \%$ formalin for $24 \mathrm{~h}$ at room temperature, embedded in paraffin and cut into $5-\mu \mathrm{m}$ sections. Following dewaxing (with xylene twice for $10 \mathrm{~min}$ ), rehydration (with $100 \%$ ethanol twice for $5 \mathrm{~min}, 95 \%$ ethanol twice for $2 \mathrm{~min}$, and $85 \%$ ethanol twice for $2 \mathrm{~min}$ at room temperature) and antigen retrieval (with citrate buffer at $98^{\circ} \mathrm{C}$ for $15 \mathrm{~min}$ ), the endogenous peroxidase activity (with $3 \%$ hydrogen peroxide for $10 \mathrm{~min}$ at room temperature) of each section was quenched. Each slide was incubated with the primary antibody (rabbit polyclonal antibody against JMJD6; 1:250 dilution; cat no. ab96795; Abcam) overnight at $4^{\circ} \mathrm{C}$. Subsequently, the samples were incubated with a biotinylated secondary antibody for $50 \mathrm{~min}$ at $37^{\circ} \mathrm{C}$, and stained with a $3^{\prime}$-diaminobenzidine kit (GT Vision Ltd.) for $1 \mathrm{~min}$ at room temperature. Consecutive sections were counterstained with hematoxylin and eosin (hematoxylin staining for $5 \mathrm{~min}$ and $0.5 \%$ eosin staining for $1 \mathrm{~min}$, at room temperature).

The immunoreactivity score of JMJD6 staining was recorded by two independent observers under a light microscope (Olympus Corporation) at x100 and x200 magnification, according to staining intensity and percentage of positive cancer cells. The staining intensity was scored as follows: Weak, scored 1; moderate, scored 2; and intense, scored 3. For the percentage of positive cancer cells, the score was defined as follows: $\leq 25 \%$, scored $1 ;>25 \%, \leq 50 \%$, scored 2 ; $>50, \leq-75 \%$, scored $3 ;>75 \%$, scored 4 . Finally, a total score (ranging between 1 and 12) was acquired by multiplying the scores for staining intensity and for the percentage of positive cancer cells. A score of 1-6 was considered to indicate low expression, whereas a score of 7-12 was considered to indicate high expression.

Cell culture and plasmid transfection. The Cal27 and HN12 HNSCC cell lines were cultured in DMEM (Shanghai Basal Media Technologies Co., Ltd.) supplemented with 10\% FBS (Gibco; Thermo Fisher Scientific, Inc.), $100 \mathrm{U} / \mathrm{ml}$ penicillin and $100 \mu \mathrm{g} / \mathrm{ml}$ streptomycin at $37^{\circ} \mathrm{C}$ with $5 \% \mathrm{CO}_{2}$.

Cal27 cells function as a classical HNSCC cell line, and HN12 cells possess an increased epithelial-mesenchymal transition (EMT) potential. In the present study, JMJD6 was overexpressed in Cal27 cells and knocked down in HN12 cells. The mammalian expression vector used was pcDNA3.1(+)-myc-JMJD6 and pcDNA3.1(+) was used as an empty vector control (Genomeditech). The plasmids $(2 \mu \mathrm{g})$ were transfected into cells using Lipofectamine ${ }^{\circledR}$ 2000 (Invitrogen; Thermo Fisher Scientific, Inc.) diluted in OPTI-MEM $^{\circledR}$ I (Thermo Fisher Scientific, Inc.) according to the manufacturer's protocol. Small interfering RNAs (siRNAs) were constructed (Genomeditech) and validated at the mRNA and protein levels. Validated small interfering RNAs (siRNAs, $50 \mathrm{nM}$ ) specific to JMJD6 were transfected into HN12 cells. The sequence of the siRNA targeting JMJD6 was 5'-GAG GGAACCAGCAAGACGA-3', and the sequence for the scrambled siRNA was 5'-UUCUCCGAACGUGUCACGUdT dT-3' (Genomeditech, Co., Ltd.). For the subsequent analysis, RNA was extracted after $24 \mathrm{~h}$ transfection, and protein was extracted $48 \mathrm{~h}$ after transfection.

Western blot analysis. Cellular extracts were acquired by using whole-cell lysis buffer (Yeasen Biotechnology, Co., Ltd.) containing a proteinase inhibitor cocktail (1 $\mathrm{mM}$ PMSF, Yeasen Biotechnology, Co., Ltd.; cat. no. 20104ES03). The protein concentration was determined using BCA assays (Thermo Fisher Scientific, Inc.). After subjecting the lysates (30 $\mu \mathrm{g}$ protein per lane) to $10 \%$ SDS-PAGE, proteins were transferred to a PVDF membrane by electroblotting. Subsequently, the membranes were blocked in 5\% skimmed milk for $1 \mathrm{~h}$ at room temperature and incubated overnight at $4^{\circ} \mathrm{C}$ with specific primary antibodies against JMJD6 (1:1,000; cat. no. ab50720), Snai1 (1:1,000; cat. no. ab53519), Vimentin (1:1,000; cat. no. ab16700), Twist1 (1:1,000; cat. no. ab175430), N-cadherin (1:1,000; cat. no. ab98952), E-cadherin (1:1,000; cat. no. ab40772) (all from Abcam) and GAPDH (1:5000; cat. no. BM3876; Wuhan Boster Biological Technology, Ltd.). Specific secondary antibodies (horseradish peroxidase-conjugated goat anti-mouse IgG or horseradish peroxidase-conjugated goat anti-rabbit IgG; both 1:5,000; cat. nos. 33201ES60 or 34301ES60, respectively; both from Yeasen Biotechnology, Co., Ltd.;) were incubated in room temperature for $1 \mathrm{~h}$. Specific antibody-bound protein bands were detected with ECL Plus reagent (EMD Millipore) using an Amersham Imager 600 (GE Healthcare).

Reverse transcription-quantitative PCR. Total RNA was extracted from treated HNSCC cells using lysis buffer (Takara Bio, Inc.) according to the manufacturer's protocol. Then, 
Table I. Primer sequences for each gene.

Gene name

\section{JMJD6}

SNAII

VIM

TWIST1

$\mathrm{CDH} 1$

$\mathrm{CDH} 2$

GAPDH
Forward primer (5'-3')

Reverse primer (5'-3')

CDH1, cadherin 1; CDH2, cadherin 2; JMJD6, Jumonji domain-containing protein 6; SNAI1, snail family transcriptional repressor 1; TWIST1, twist family bHLH transcription factor 1 ; VIM, vimentin.

reverse transcription was performed by using the PrimeScript RT reagent kit (Takara Bio, Inc.) with the following RT protocol: $15 \mathrm{~min}$ at $37^{\circ} \mathrm{C}$ and $5 \mathrm{sec}$ at $85^{\circ} \mathrm{C}$. The cDNA was subjected to PCR detection using a SYBR Green Premix kit (Takara Bio, Inc.) with the following protocols: $95^{\circ} \mathrm{C}$ for $30 \mathrm{sec}$; and followed by 40 cycles, $95^{\circ} \mathrm{C}$ for $5 \mathrm{sec}$ and $60^{\circ} \mathrm{C}$ for $34 \mathrm{sec}$. Relative expression was calculated using the $2^{-\Delta \Delta \mathrm{Cq}}$ method by using GAPDH as reference (15). The primers for each gene are listed in Table I.

Transwell assays. For cellular migration, $2 \times 10^{4}$ cells suspended in $200 \mu \mathrm{l}$ fresh DMEM were plated in Millicell chambers (8- $\mu \mathrm{m}$ pore size; EMD Millipore), and $600 \mu \mathrm{l}$ DMEM containing 10\% FBS was plated in the lower chamber. Cells that migrated through the membrane were fixed with $4 \%$ paraformaldehyde for $15 \mathrm{~min}$ at room temperature and stained with $0.1 \%$ crystal violet for $15 \mathrm{~min}$ at room temperature. For cellular invasion assays, the chamber was coated with $50 \mu \mathrm{l}$ Matrigel for $1 \mathrm{~h}$ at room temperature, which was then hydrated for $2 \mathrm{~h}$ at $37^{\circ} \mathrm{C}$ (1:8 dilution; BD Biosciences) prior to the assay. Images of the membrane were captured and the number of migratory or invasive cells was observed under a light microscope (Olympus Corporation) and counted from five fields of view (magnification, x100).

Colony formation assay. A cellular suspension was diluted and seeded at $1 \times 10^{3}$ cells/well on 6 -well plates in triplicate. The cells were cultured with culture medium (DMEM supplemented with $10 \% \mathrm{FBS}, 100 \mathrm{U} / \mathrm{ml}$ penicillin and $100 \mu \mathrm{g} / \mathrm{ml}$ streptomycin) for 10 days, and then harvested and fixed with $4 \%$ paraformaldehyde for $15 \mathrm{~min}$ at room temperature. The cell colonies were observed by staining with Coomassie brilliant blue for $30 \mathrm{~min}$ at room temperature. The number of large colonies (consisting of $\geq 50$ cells) was counted and analyzed under a light microscope, at x100 magnification (Olympus Corporation).

Cell viability analysis. MTT assays were performed to examine the viability of cells. Briefly, cells were diluted and seeded at $1 \times 10^{4}$ cells $/ 100 \mu \mathrm{l}$ in 96 -well plates for $12 \mathrm{~h}$ for attachment, and then incubated with 5-fluorouracil (5-FU; Selleck Chemicals) or cisplatin (Selleck Chemicals) at various concentrations for $48 \mathrm{~h}$ at $37^{\circ} \mathrm{C}$ with $5 \% \mathrm{CO}_{2}$. Absorbance measurements following DMSO resolution were performed at a wavelength of $490 \mathrm{~nm}$ (Bio-Rad Laboratories, Inc.).

Statistical analysis. All statistical analyses were conducted using SPSS v16.0 software (SPSS, Inc.). Statistical comparisons of the transcriptional level of JMJD6 among different stages were made using one-way ANOVA and least significant difference post hoc test (LSD). The different Kaplan-Meier curves were compared by using the log-rank test. Bioinformatically, based on the detailed data of each biomarker, all cases involved were divided equally into two groups; the high-expression group and the low-expression group (the median expression levels were used as cut-off values for each biomarker: 9.318 for JMJD6, 6.433 for SNAI1, 13.53 for vimentin, 7.299 for Twist1, 5.103 for $\mathrm{CDH} 2$, and 13.23 for CDH1). To illustrate the underlying association between JMJD6 and EMT markers, crosstabs analyses were performed, and the $\chi^{2}$ test and Pearson's R correlation coefficient test were used to assess the associations between the gene expression levels of JMJD6 and the gene expression levels of EMT markers (16). Significant differences between two groups were determined using unpaired Student's t-test. The $\mathrm{IC}_{50}$ values were calculated with GraphPad Prism (version 6; GraphPad Software, Inc.). All experiments were performed in triplicate and representative results are presented. The values presented correspond to the mean \pm standard deviation. $\mathrm{P}<0.05$ was considered to indicate a statistically significant difference.

\section{Results}

JMJD6 is highly expressed in HNSCC tissues. Based on the bioinformatics results from GEPIA2, the mRNA expression levels of JMJD6 were significantly upregulated in HNSCC tissues compared with normal tissues in the head and neck area (Fig. 1A and B). However, when further prognostic analysis was performed, no obvious significant differences were observed in overall survival (OS) or disease-free survival (DFS) times in HNSCC for different JMJD6 expression levels (Fig. 1C and D). Therefore, the exact roles of highly expressed JMJD6 in HNSCC remain to be determined. In the present study, bioinformatics analysis based on TCGA HNSCC database revealed a significant association between the expression level of JMJD6 and the pathological stage of HNSCC (Fig. 1E; Table II). These 
A

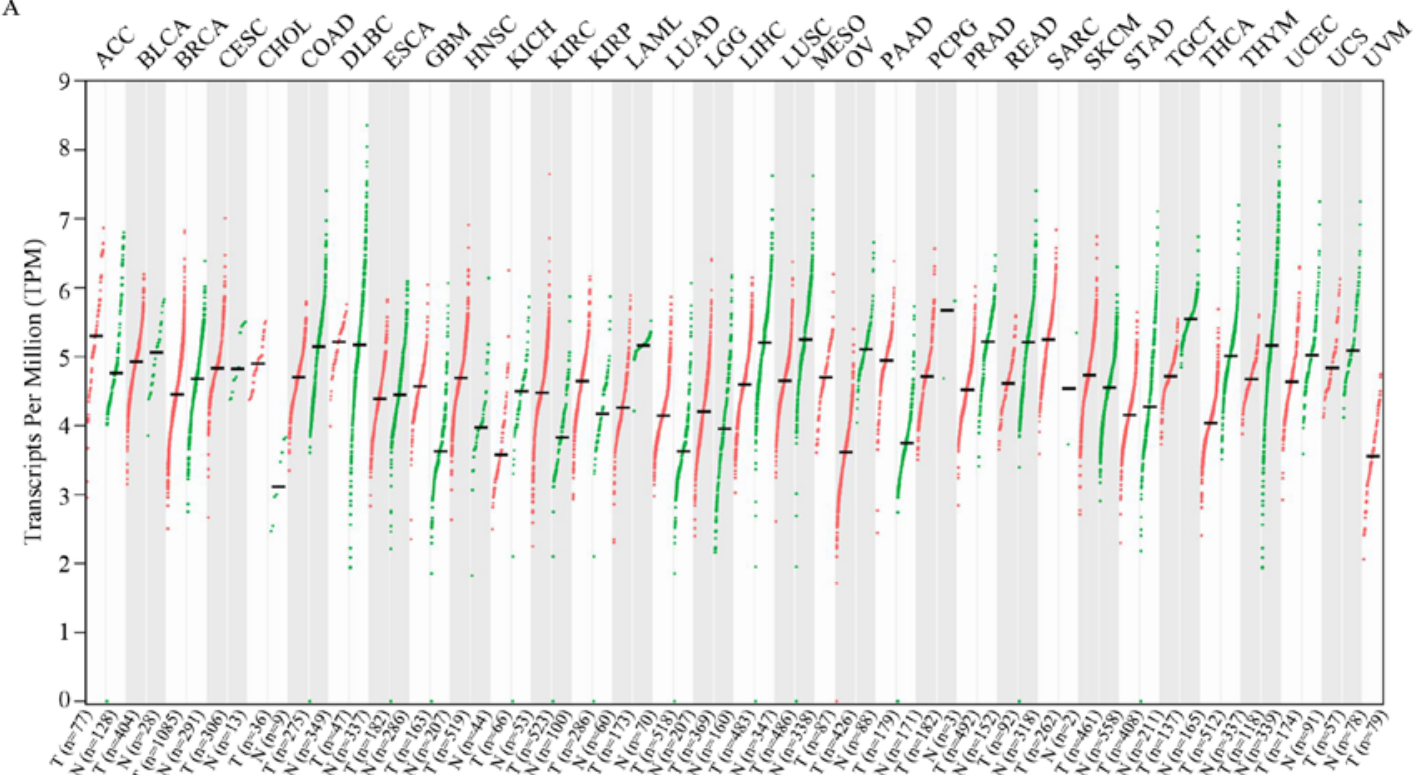

B

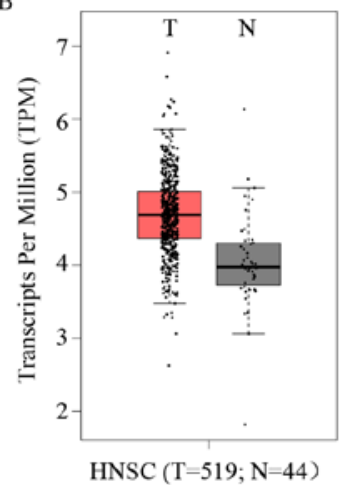

C

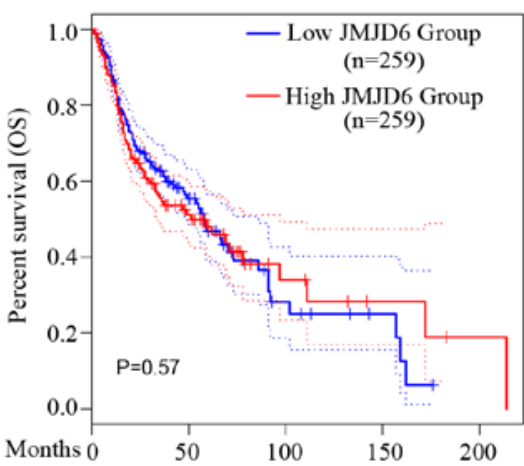

D

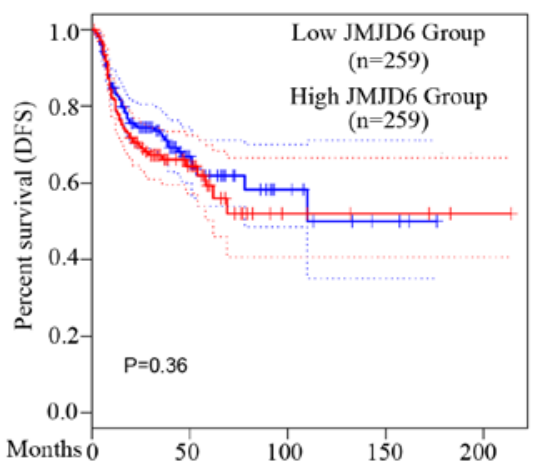

E

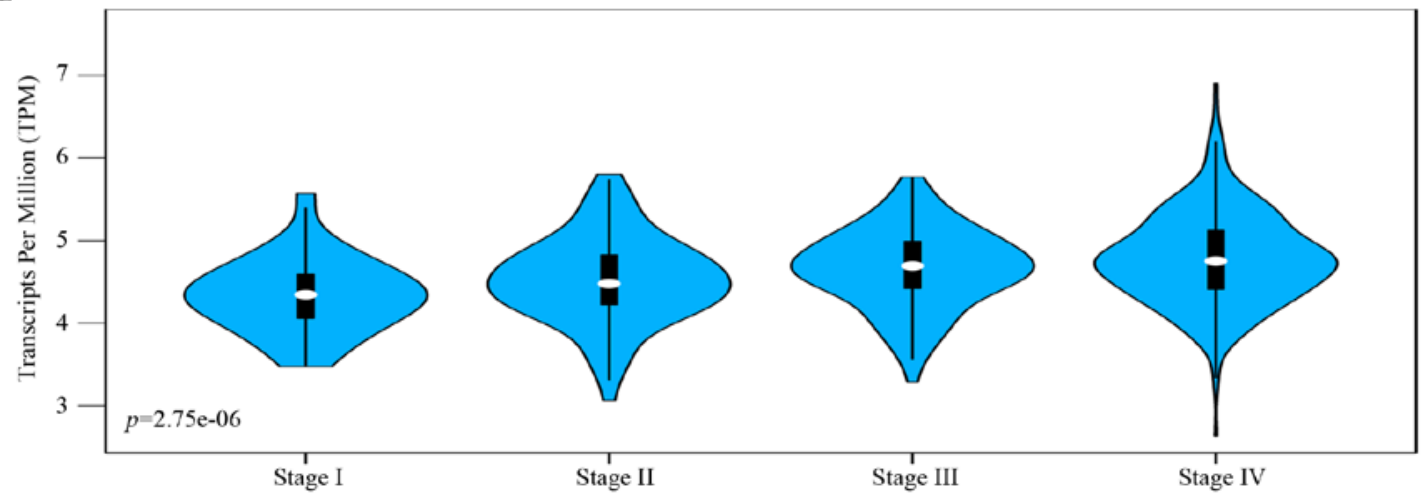

Figure 1. Bioinformatics analysis of the clinical significance of JMJD6 in HNSCC. (A) Expression patterns of JMJD6 in different types of cancer. (B) Expression pattern of JMJD6 in HNSCC. Kaplan-Meier curves for (C) OS time and (D) DFS time analyses, based on JMJD6 expression in HNSCC. (E) Schematic diagram of the association between JMJD6 expression and pathological stage in HNSCC (** $<<0.01)$. JMJD6, Jumonji domain-containing protein 6; HNSCC, head and neck squamous cell carcinoma, T, tumor; N, normal; OS, overall survival; DFS, disease-free survival.

results indicated that JMJD6 could predict HNSCC cases with an advanced stage but had no predictive value for prognosis.

High JMJD6 expression acts as a risk factor for the malignant progression of patients with HNSCC. Based on 566 cases from the TCGA primary HNSCC cohort, increased JMJD6 expression was significantly associated with pathologic tumor size, pathologic lymph node status, pathologic stage and pathological nodal extracapsular spread (the AJCC Cancer Staging manual for head and neck cancer 8th Edition, 2017) (17) (Table II). In the retrospectively validation cohort, based on 98 HNSCC cases, all cases were divided into two group under the expression level of JMJD6 (Fig. 2). Besides, significant associations between increased JMJD6 expression and tumor size and nodal status were observed (Table III). These results suggested that JMJD6 may promote the malignant progression of HNSCC. 
Table II. Pathological characteristics of head and neck squamous cell carcinoma cases, based on The Cancer Genome Atlas cohort, according to JMJD6 expression.

\begin{tabular}{|c|c|c|c|c|}
\hline \multirow[b]{2}{*}{ Variables } & \multirow[b]{2}{*}{ Patients, $\mathrm{n}$} & \multicolumn{2}{|c|}{ JMJD6 expression } & \multirow[b]{2}{*}{ P-value } \\
\hline & & $\leq 9.318, \mathrm{n}$ & $>9.318, \mathrm{n}$ & \\
\hline All cases & 566 & 283 & 283 & \\
\hline Pathologic T & & & & 0.005 \\
\hline $\mathrm{T} 1+\mathrm{T} 2$ & 208 & 119 & 89 & \\
\hline $\mathrm{T} 3+\mathrm{T} 4$ & 295 & 131 & 164 & \\
\hline Pathologic N & & & & 0.013 \\
\hline No & 193 & 107 & 86 & \\
\hline $\mathrm{N} 1+$ & 260 & 113 & 147 & \\
\hline Pathologic M & & & & I \\
\hline M0 & 190 & 85 & 105 & \\
\hline M1 & 1 & 1 & 0 & \\
\hline Pathologic stage & & & & $<0.001$ \\
\hline $\mathrm{I}+\mathrm{II}$ & 119 & 77 & 42 & \\
\hline III+IV & 374 & 167 & 207 & \\
\hline Neoplasm histologic grade & & & & 0.143 \\
\hline $\mathrm{G} 1+\mathrm{G} 1$ & 398 & 206 & 192 & \\
\hline $\mathrm{G} 3+\mathrm{G} 4$ & 142 & 63 & 79 & \\
\hline Lymphovascular invasion present & & & & 0.052 \\
\hline Yes & 136 & 54 & 82 & \\
\hline No & 239 & 121 & 118 & \\
\hline Pathological nodal extracapsular spread & & & & $<0.001$ \\
\hline Yes & 118 & 36 & 82 & \\
\hline No & 262 & 136 & 126 & \\
\hline Perineural invasion present & & & & 0.613 \\
\hline Yes & 186 & 83 & 103 & \\
\hline No & 208 & 99 & 109 & \\
\hline
\end{tabular}

Some clinicopathological parameters had missing data and so were excluded. JMJD6, Jumonji domain-containing protein 6; /, not applicable.

JMJD6 regulates the EMT behavior of HNSCC. JMJD6 has been demonstrated to induce EMT and promote malignant migration and invasion in breast cancer (18). In the present study, bioinformatics analysis based on the TCGA primary HNSCC cohort was performed to analyze the association between JMJD6 expression and EMT status (based on the expression of SNAI1, VIM, TWIST1, CDH2 and CDH1). As shown in Fig. 3A, significant positive correlations between JMJD6 and SNAI1, TWIST1 and CDH2 expression and a significant negative correlation between JMJD6 and $\mathrm{CDHI}$ expression were observed. Subsequently, a JMJD6 overexpression model in Cal27 cells and a JMJD6 knockdown model in $\mathrm{HN} 12$ cells were constructed (Fig. 3B). Overexpression of JMJD6 in Cal27 cells resulted in increased expression levels of SNAI1, VIM, TWIST1 and CDH2, and significantly decreased the expression levels of $\mathrm{CDH}$ (Fig. 3B and D). Similarly, JMJD6 was knocked down in HN12 cells, which resulted in decreased expression levels of SNAI1, VIM, TWISTI and $\mathrm{CDH}$, while significantly increased expression levels of $\mathrm{CDH} 1$ were observed (Fig. 3C and D). Morphologically, Cal27 cells exhibited an epithelial multiangle shape, and a long, spindle shape was observed in Cal27 cells overexpressing JMJD6 (Fig. 3E). In HN12 cells, knockdown of JMJD6 resulted in a morphological alteration from a long, spindle shape to a multiangle shape (Fig. 3E). Therefore, increased JMJD6 expression could regulate the EMT status of HNSCC cells.

JMJD6 overexpression promotes the colony formation, and migration and invasion abilities of HNSCC cells. Clinicopathological analysis based on the TCGA primary HNSCC cohort and the validation cohort indicated roles of JMJD6 in the regulation of the metastatic ability of HNSCC. Furthermore, additional roles of JMJD6 in the regulation of EMT were demonstrated in the present study. Colony formation assays indicated markedly increased colony-forming abilities in JMJD6-overexpression Cal27 cells, whereas the knockdown of JMJD6 in HN12 cells significantly decreased their colony-forming abilities (Fig. 4A and B). Transwell assays were performed to investigate the effects of JMJD6 on the migration and invasion ability of HNSCC cells. Overexpression of 
Table III. Clinicopathological characteristics of patients divided by JMJD6 expression based on a validation head and neck squamous cell carcinoma cohort.

JMJD6 expression

\begin{tabular}{|c|c|c|c|c|}
\hline \multirow[b]{2}{*}{ Variable } & \multirow[b]{2}{*}{ Patients, $\mathrm{n}$} & & \multirow[b]{2}{*}{ P-value } \\
\hline & & $\mathrm{IRS}=1-6, \mathrm{n}$ & $\mathrm{IRS}=7-12, \mathrm{n}$ & \\
\hline All cases & 98 & 48 & 50 & \\
\hline Age, years & & & & 0.837 \\
\hline$<60$ & 59 & 28 & 31 & \\
\hline$\geq 60$ & 39 & 20 & 19 & \\
\hline Sex & & & & 0.843 \\
\hline Male & 52 & 26 & 26 & \\
\hline Female & 46 & 22 & 24 & \\
\hline Smoking & & & & 0.999 \\
\hline Yes & 51 & 25 & 26 & \\
\hline No & 47 & 23 & 24 & \\
\hline Alcohol & & & & 0.999 \\
\hline Yes & 48 & 24 & 24 & \\
\hline No & 50 & 24 & 26 & \\
\hline Histological grade & & & & 0.110 \\
\hline Well/moderate-differentiation & 53 & 30 & 23 & \\
\hline Poor-differentiation & 45 & 18 & 27 & \\
\hline Tumor size & & & & 0.017 \\
\hline $\mathrm{T} 1+\mathrm{T} 2$ & 51 & 31 & 20 & \\
\hline $\mathrm{T} 3+\mathrm{T} 4$ & 47 & 17 & 30 & \\
\hline Nodal status & & & & 0.003 \\
\hline No & 52 & 33 & 19 & \\
\hline $\mathrm{N} 1+$ & 46 & 15 & 31 & \\
\hline Metastasis & & & & I \\
\hline M0 & 98 & 48 & 50 & \\
\hline M1 & 0 & 0 & 0 & \\
\hline
\end{tabular}

IRS, immunoreactivity score; JMJD6, Jumonji domain-containing protein 6; /, not applicable.
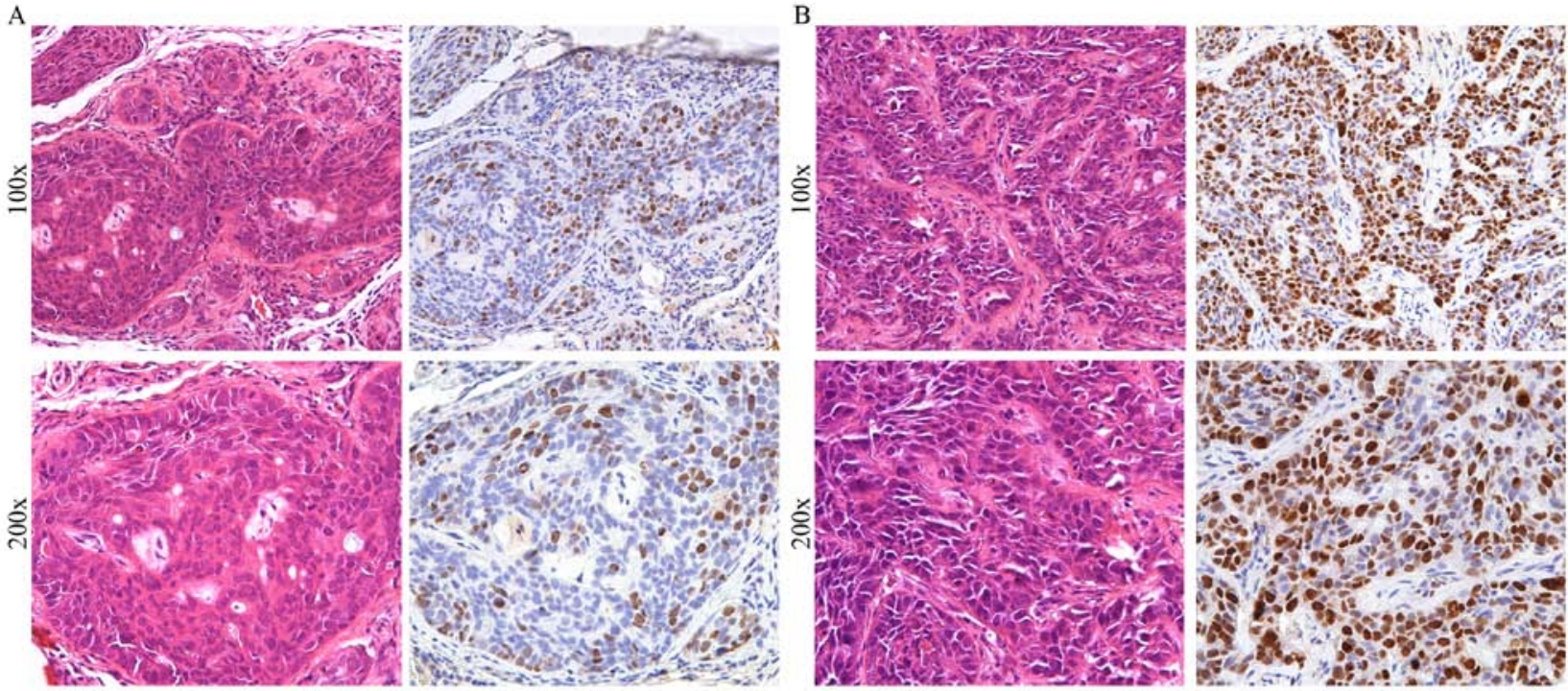

Figure 2. Representative hematoxylin and eosin staining, and JMJD6 immunohistochemical staining of consecutive sections of head and neck squamous cell carcinoma cases. Representative staining for cases with (A) low and (B) high JMJD6 expression. JMJD6, Jumonji domain-containing protein 6. 
A

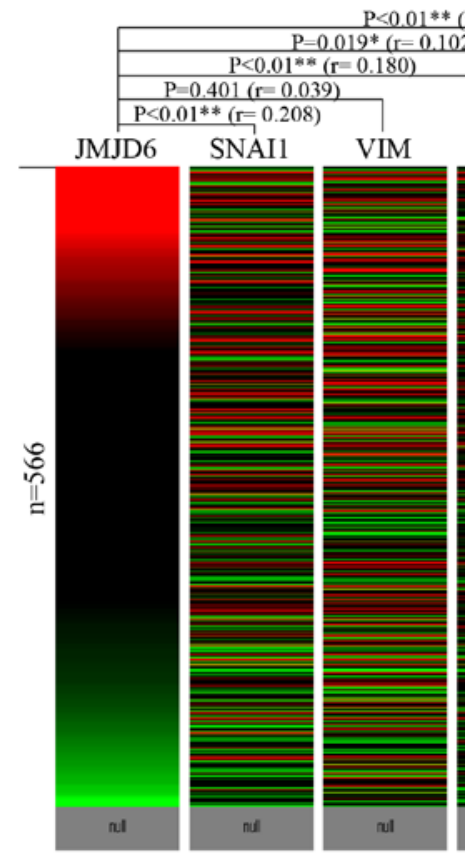

D

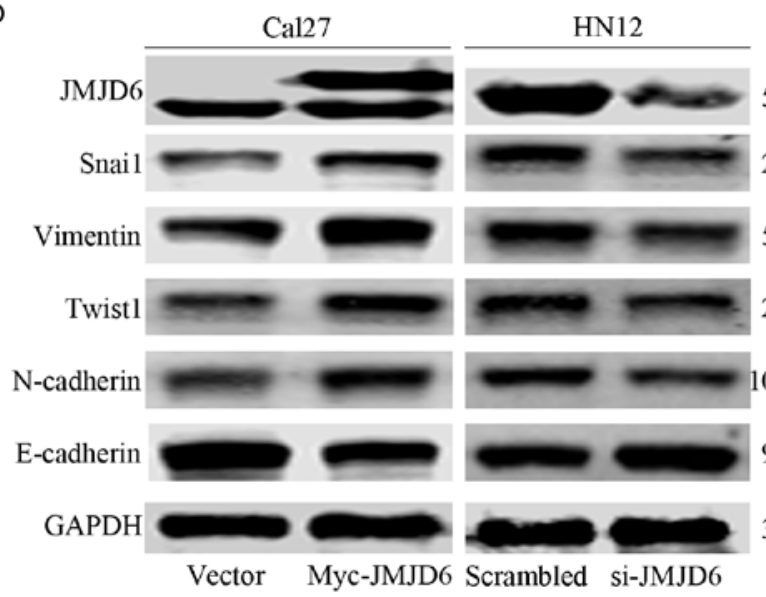

B

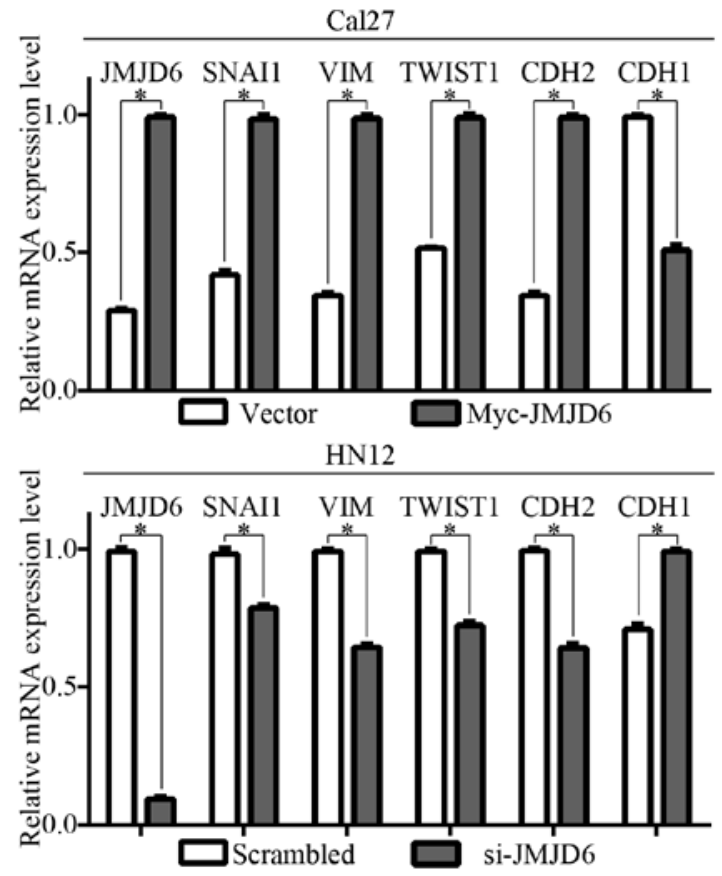

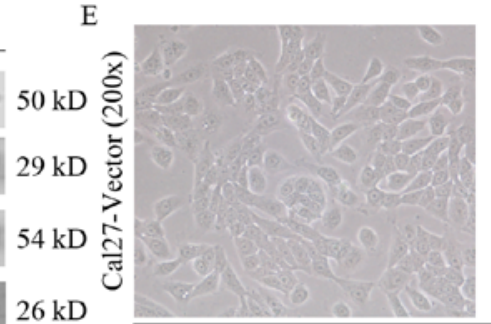

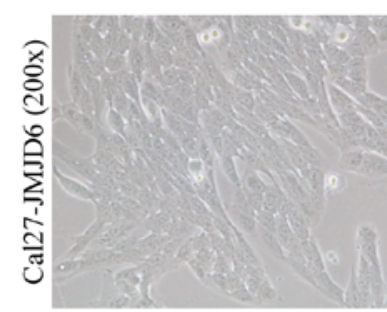

$100 \mathrm{kD}$ 旁

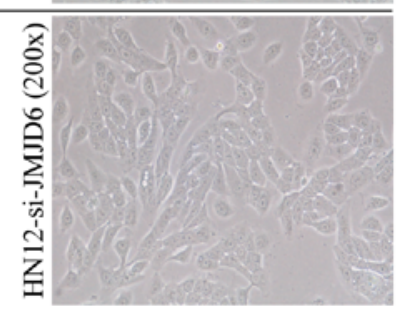

Figure 3. Effects of JMJD6 expression on the epithelial-mesenchymal transition status of HNSCC. (A) Heat map from the UCSC Xena Browser, based on the primary HNSCC cohort from The Cancer Genome Atlas revealing the associations between JMJD6 and SNAI1, VIM, TWIST1, CDH2 and CDH1 gene expression. B A significant correlation with JMJD6 expression was observed for SNAII, TWISTI (Positive correlation) and CDHI (negative correlation). (B) mRNA expression levels of JMJD6, SNAI1, VIM, TWIST1, CDH2 and CDH1 in Cal27 cells transfected with Myc-JMJD6 for $72 \mathrm{~h}$. (C) mRNA expression levels of JMJD6, SNAII, VIM, TWIST1, CDH2 and CDH1 in HN12 cells transfected with siRNA-JMJD6 for $72 \mathrm{~h}$. (D) Western blot analysis of the expression of JMJD6 in transiently transfected Cal27 and HN12 cells. The upper band of JMJD6 for Cal27 cells indicated the exogenous myc-JMJD6. (E) Representative morphological images of Cal27 cells transfected with Myc-JMJD6 and HN12 cells transfected with si-JMJD6 for 4 days. Magnification, x200. "P<0.05. ${ }^{* *} \mathrm{P}<0.01$. CDH1, cadherin 1; $\mathrm{CDH} 2$, cadherin 2; JMJD6, Jumonji domain-containing protein 6; HNSCC, head and neck squamous cell carcinoma; siRNA, small interfering RNA; SNAI1, snail family transcriptional repressor 1; TWIST1, twist family bHLH transcription factor 1; VIM, vimentin.

JMJD6 in Cal27 cells resulted in significantly increased migration and invasion (Fig. 4C and D). By contrast, knockdown of JMJD6 in HN12 cells resulted in significantly decreased migration and invasion (Fig. 4C and D).

JMJD6 has no effect on the sensitivity of HNSCC to drugs. The aforementioned data indicated that JMJD6 is significantly associated with malignant behaviors but not with the clinical outcomes of HNSCC. The viability of Cal27 and HN12 cells with different JMJD6 expression levels in response to treatment with 5-FU or cisplatin was investigated, and the $\mathrm{IC}_{50}$ values were calculated. No obvious differences were observed in the $\mathrm{IC}_{50}$ values of HNSCC cells treated with 5-FU or cisplatin, in association with the different expression levels of JMJD6 (Fig. 5A and B). Therefore, HNSCC with high JMJD6 expression may exhibit a more advanced stage; however, to the best of our knowledge, this is not associated with responsiveness to chemotherapy.

\section{Discussion}

The dysregulation of JMJD6 has been reported to contribute to several types of human cancer by controlling a wide range of biological functions $(3,4)$. To the best of our knowledge, the 
A

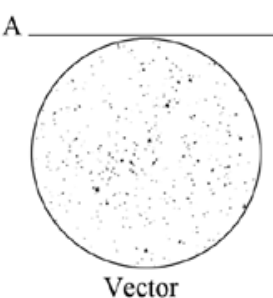

$\mathrm{C}$

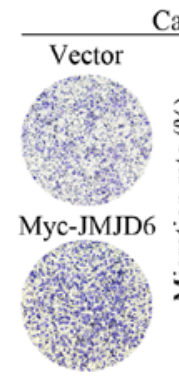

Cal27
$\mathrm{Cal} 27$
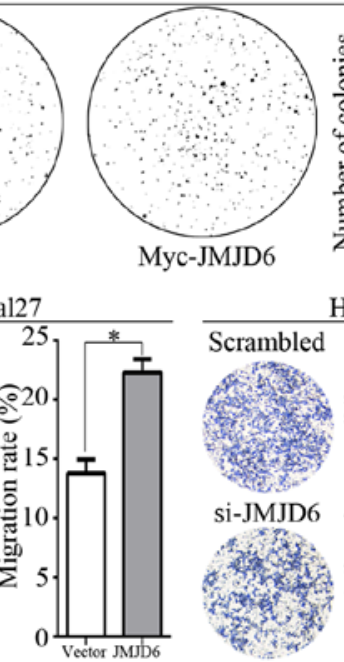

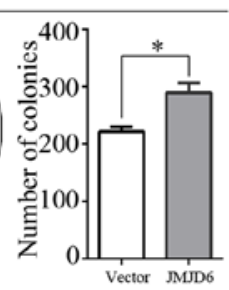

HN12

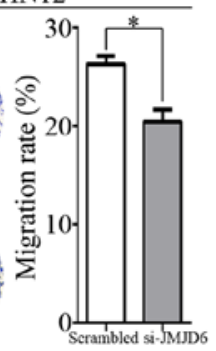

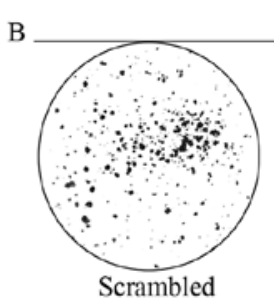

D

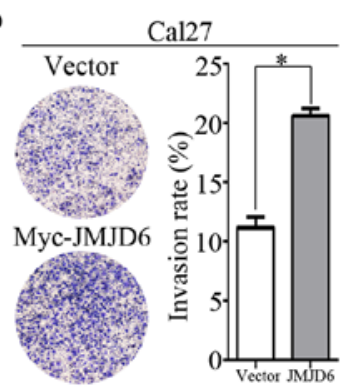

HN12

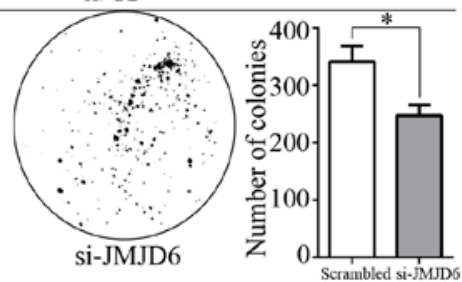

$\mathrm{HN} 12$

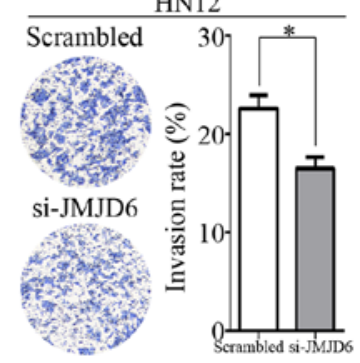

Figure 4. Effects of JMJD6 expression on the behaviors of head and neck squamous carcinoma cells. Colony-formation assay in (A) Cal27 cells transfected with Myc-JMJD6 and (B) HN12 cells transfected with si-JMJD6. (C) Transwell assays for evaluating the effects of JMJD6 overexpression on the migration ability of Cal27 cells and the effects of JMJD6 knockdown on the migration ability of HN12 cells. (D) Transwell assays for evaluating the effects of JMJD6 overexpression on the invasion ability of Cal27 cells and the effects of JMJD6 knockdown on the invasion ability of HN12 cells. Three independent experiments were performed and representative images are shown. ${ }^{*} \mathrm{P}<0.05$. JMJD6, Jumonji domain-containing protein 6; si, small interfering RNA.
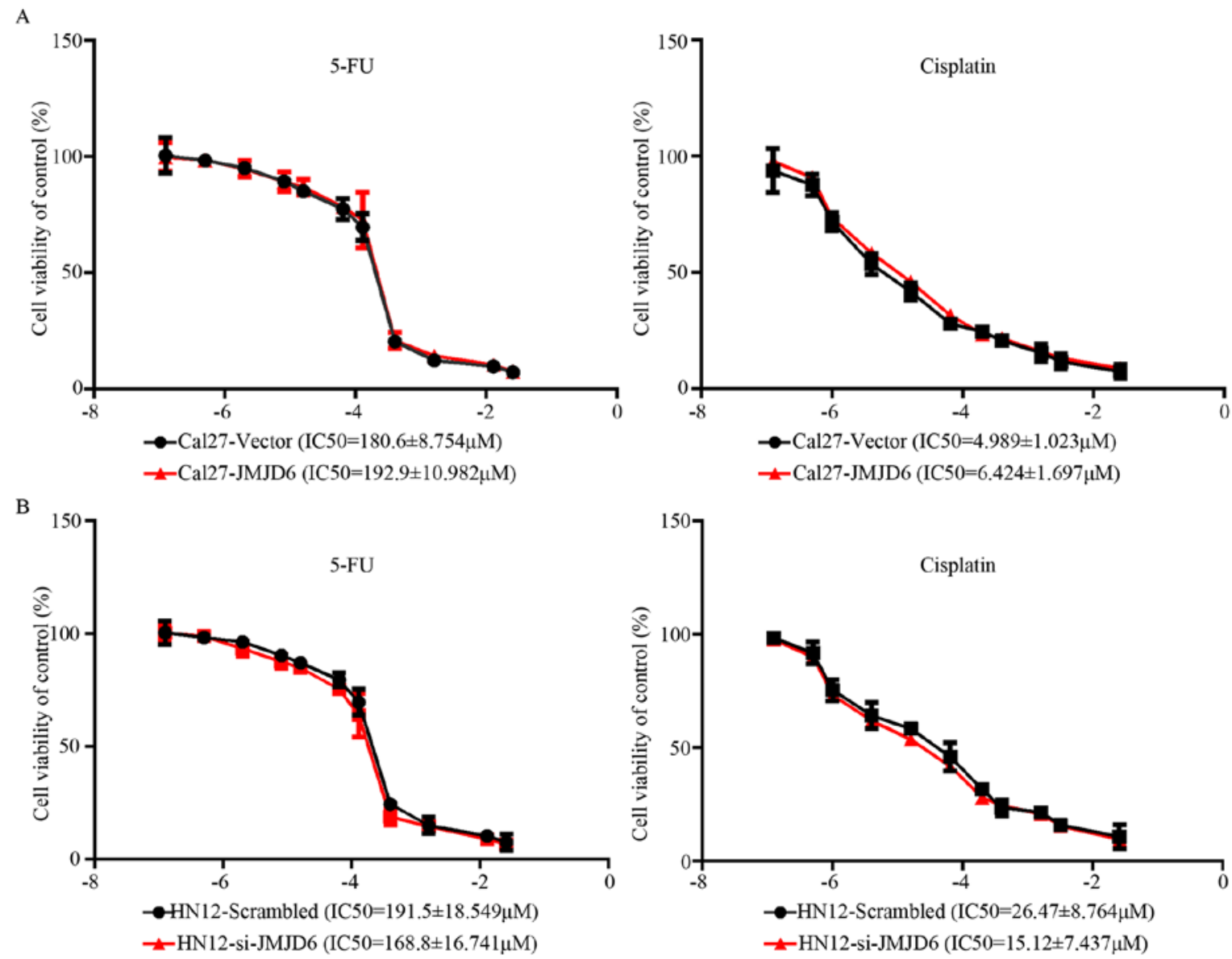

Figure 5. Effects of JMJD6 expression on the drug responses of head and neck squamous cell carcinoma cells. Determination of $\mathrm{IC}_{50}$ of $(\mathrm{A})$ Cal27 cells following JMJD6 overexpression and (B) HN12 cells following JMJD6 knockdown in response to 5-FU and cisplatin. 5-FU, 5-fluorouracil; JMJD6, Jumonji domain-containing protein 6; si, small interfering RNA.

biological roles and clinical significance of JMJD6 in HNSCC remain uncertain. In the present study, the clinical significance of JMJD6 based on the TCGA primary HNSCC cohort and a validation HNSCC cohort was investigated. Subsequently, 
the roles of JMJD6 in HNSCC were revealed through a series of cellular assays. Consequently, it can be concluded that JMJD6 contributes to the malignant progression of HNSCC by regulating EMT, whereas JMJD6 overexpression exerts no significant effects on the drug responsiveness and prognosis of HNSCC.

The data from the present study demonstrated that JMJD6 was significantly upregulated in HNSCC samples compared with normal samples. Overexpression of JMJD6 did not affect the prognosis (OS and DFS times) of patients with HNSCC; however, it was strongly associated with advanced pathological stage (particularly in terms of tumor size, nodal status and nodal extracapsular spread), based on the TCGA cohort and a validation cohort. Previous studies have demonstrated that the upregulation of JMJD6 indicates aggressive phenotypes and a poor prognosis in multiple types of human cancer $(3,11)$. The present study suggested that patients with HNSCC and high JMJD6 expression are liable to develop advanced-stage disease but exhibited no difference in drug sensitivity. Further studies are required to analyze the expression of JMJD6 across different HNSCC subtypes.

Based on the clinical significance of JMJD6, it was speculated that JMJD6 serves key roles in the regulation of migration/invasion behaviors in HNSCC. Invasion and migration are the main characteristics of the EMT process (19). In the present study, bioinformatics analysis based on the TCGA primary HNSCC cohort was carried out in order to detect the association between JMJD6 expression and EMT status (based on the expression of SNAI1, VIM, TWIST1, $\mathrm{CDH} 2$ and $\mathrm{CDH1}$ ). As expected, JMJD6 was associated with EMT status in HNSCC. Additionally, overexpression of JMJD6 in HNSCC cells resulted in increased migration and invasion rates. Thus, JMJD6 promoted the malignant progression of HNSCC by regulating the EMT process. In lung cancer, JMJD6 has been reported to cooperate with c-Myc to enhance the EMT process (18). In HNSCC, the underlying mechanisms will be further investigated in subsequent studies.

Based on the results of IC50 experiments, the present study revealed that overexpression of JMJD6 had no effect on the drug responses of HNSCC to chemotherapy. These data may illustrate that JMJD6 contributed to a more advanced stage, however not a poorer prognosis. To the best our knowledge, the present study was the first to demonstrate that high JMJD6 expression promoted malignant progression but not the development of drug resistance in HNSCC. Treatment strategies for HNSCC with an advanced stage should be based on combined and sequential therapy. For patients with advanced HNSCC, radical surgeries always lead to poor quality of life without a markedly improved prognosis (20). According to the data obtained in the present study, rational chemotherapy may be beneficial for patients with advanced HNSCC who exhibit high JMJD6 expression.

In conclusion, the present study demonstrated the clinical significance of JMJD6 in HNSCC, and that high JMJD6 expression was significantly associated with an advanced stage, with no effect on drug responsiveness or prognosis. However, additional studies are required to investigate the underlying mechanisms in the future.

\section{Acknowledgements}

Not applicable.

\section{Funding}

The present study was supported by the Excellent Young Doctor Scholarship of the Shanghai 9th People's Hospital (grant no. 20170912) and the college fund of Shanghai Jiao Tong University School of Medicine (grant no. 13XJ10049).

\section{Availability of data and materials}

The datasets analysed during the present study are available in the TCGA-HNSC repository (https://xenabrowser.net), or available from the corresponding author upon reasonable request.

\section{Authors' contributions}

BG, YS and CM conceived and designed the study. BG and LW performed the experiments. BG and XQ compiled and analyzed the data. BG, LW and XQ contributed to writing and revising the paper. All authors read and approved the final manuscript.

\section{Ethics approval and consent to participate}

All patients involved in the present study provided written informed consent, and the study was approved by the Medical Ethics Committee of the 9th People's Hospital, Shanghai Jiao Tong University School of Medicine (Shanghai, China).

\section{Patient consent for publication}

Not applicable.

\section{Competing interests}

The authors declare that they have no competing interests.

\section{References}

1. Kwok J, O'Shea M, Hume DA and Lengeling A: JMJD6, a JMJC dioxygenase with many interaction partners and pleiotropic functions. Front Genet 8: 32, 2017.

2. Wang F, He L, Huangyang P, Liang J, Si W, Yan R, Han X, Liu S, Gui B, Li W, et al: JMJD6 promotes colon carcinogenesis through negative regulation of $\mathrm{p} 53$ by hydroxylation. PLoS Biol 12: e1001819, 2014

3. Liu Y, Long YH, Wang SQ, Zhang YY, Li YF, Mi JS, Yu CH, Li DY, Zhang JH and Zhang XJ: JMJD6 regulates histone H2A.X phosphorylation and promotes autophagy in triple-negative breast cancer cells via a novel tyrosine kinase activity. Oncogene 38: 980-997, 2019.

4. Wan J, Xu W, Zhan J, Ma J, Li X, Xie Y, Wang J, Zhu WG, Luo J and Zhang H: PCAF-mediated acetylation of transcriptional factor HOXB9 suppresses lung adenocarcinoma progression by targeting oncogenic protein JMJD6. Nucleic Acids Res 44: 10662-10675, 2016.

5. Unoki M, Masuda A, Dohmae N, Arita K, Yoshimatsu M, Iwai Y, Fukui Y, Ueda K, Hamamoto R, Shirakawa M, et al: Lysyl 5-hydroxylation, a novel histone modification, by Jumonji domain containing 6 (JMJD6). J Biol Chem 288: 6053-6062, 2013. 
6. Yi J, Shen HF, Qiu JS, Huang MF, Zhang WJ, Ding JC, Zhu XY, Zhou Y, Fu XD and Liu W: JMJD6 and U2AF65 co-regulate alternative splicing in both JMJD6 enzymatic activity dependent and independent manner. Nucleic Acids Res 45: 3503-3518, 2017.

7. Lee YF, Miller LD, Chan XB, Black MA, Pang B, Ong CW, Salto-Tellez M, Liu ET and Desai KV: JMJD6 is a driver of cellular proliferation and motility and a marker of poor prognosis in breast cancer. Breast Cancer Res 14: R85, 2012.

8. Konuma T, Yu D, Zhao C, Ju Y, Sharma R, Ren C, Zhang Q, Zhou MM and Zeng L: Structural mechanism of the oxygenase JMJD6 recognition by the extraterminal (ET) domain of BRD4. Sci Rep 7: 16272, 2017.

9. Wan J, Liu H, Yang L, Ma L, Liu J and Ming L: JMJD6 promotes hepatocellular carcinoma carcinogenesis by targeting CDK4. Int J Cancer 144: 2489-2500, 2019.

10. Liu X, Si W, Liu X, He L, Ren J, Yang Z, Yang J, Li W, Liu S, Pei F, Yang X and Sun L: JMJD6 promotes melanoma carcinogenesis through regulation of the alternative splicing of PAK1, a key MAPK signaling component. Mol Cancer 16: 175, 2017.

11. Lee CR, Lee SH, Rigas NK, Kim RH, Kang MK, Park NH and Shin KH: Elevated expression of JMJD6 is associated with oral carcinogenesis and maintains cancer stemness properties. Carcinogenesis 37: 119-128, 2016.

12. Zhu J, Sanborn JZ, Benz S, Szeto C, Hsu F, Kuhn RM, Karolchik D, Archie J, Lenburg ME, Esserman LJ, et al: The UCSC cancer genomics browser. Nat Methods 6: 239-240, 2009.

13. Tang Z, Li C, Kang B, Gao G, Li C and Zhang Z: GEPIA: A web server for cancer and normal gene expression profiling and interactive analyses. Nucleic Acids Res 45: W98-W102, 2017.

14. Goldman M, Craft B, Swatloski T, Cline M, Morozova O, Diekhans M, Haussler D and Zhu J: The UCSC cancer genomics browser: Update 2015. Nucleic Acids Res 43: D812-D817, 2015.
15. Livak KJ and Schmittgen TD: Analysis of relative gene expression data using real-time quantitative PCR and the 2(-Delta Delta C(T)) method. Methods 25: 402-408, 2001

16. Xiao M, Zhang J, Chen W and Chen W: M1-like tumor-associated macrophages activated by exosome-transferred THBS1 promote malignant migration in oral squamous cell carcinoma. J Exp Clin Cancer Res 37: 143, 2018.

17. Lydiatt WM, Patel SG, O'Sullivan B, Brandwein MS, Ridge JA, Migliacci JC, Loomis AM and Shah JP: Head and neck cancers-major changes in the American Joint Committee on cancer eighth edition cancer staging manual. CA Cancer J Clin 67: 122-137, 2017.

18. Aprelikova O, Chen K, El Touny LH, Brignatz-Guittard C, Han J, Qiu T, Yang HH, Lee MP, Zhu M and Green JE: The epigenetic modifier JMJD6 is amplified in mammary tumors and cooperates with c-Myc to enhance cellular transformation, tumor progression, and metastasis. Clin Epigenetics 8: 38, 2016.

19. Mittal V: Epithelial mesenchymal transition in tumor metastasis. Annu Rev Pathol 13: 395-412, 2018.

20. Miyauchi S, Kim SS, Pang J, Gold KA, Gutkind JS, Califano JA, Mell LK, Cohen EEW and Sharabi AB: Immune modulation of head and neck squamous cell carcinoma and the tumor microenvironment by conventional therapeutics. Clin Cancer Res 25: 4211-4423, 2019.

This work is licensed under a Creative Commons Attribution-NonCommercial-NoDerivatives 4.0 International (CC BY-NC-ND 4.0) License. 